\title{
Preservation of Fertility in a Patient with a Mixed Germ Tumor and Subsequent Successful Pregnancies. A Case Report
}

\author{
Otto H. Valdés-Martínez ${ }^{1 *}$, Sara P. Peña-Lizola¹, Oscar Vidal-Gutiérrez², Luis H. Sordia-Hernández ${ }^{1}$, Felipe \\ A. Morales-Martínez ${ }^{1}$ and Emilio Treviño-Salinas ${ }^{2}$ \\ ${ }^{1}$ Reproductive Medicine University Center, Mexico; ${ }^{2}$ Gynecology Service. "Dr. José Eleuterio González" University Hospital, Universidad Autónoma \\ de Nuevo León, Mexico
}

\begin{abstract}
Objective: The objective of the study was to present the case of a patient with a diagnosis of a mixed germ cell tumor clinical stage (CS) IIC, who underwent conservative cytoreductive surgery and chemotherapy before initiation of a gonadotropin-releasing hormone agonist (GnRH-a) for fertility preservation, subsequently achieving spontaneous pregnancies. Material and methods: A 19-year-old patient underwent an examination for abdominal distention. Through clinical and imaging studies, an abdominal-pelvic mass of $15 \mathrm{~cm} \times 14 \mathrm{~cm} \times 10 \mathrm{~cm}$ was diagnosed with a location in the right ovary. After optimal cytoreductive surgery, the report confirmed a conservative germinal mixed tumor histopathology ( $80 \%$ endodermic sinuses and $20 \%$ embryonal carcinoma), CS IIC, so 4 cycles of chemotherapy were indicated. Before the start of and throughout the cytotoxic treatment, GnRH-a was administered. Results: At the end of the oncological treatment, a follicle stimulating hormone of $20 \mathrm{mUI} / \mathrm{mL}$ was reported, spontaneous resumption of menstrual cycles was presented 2 months after having concluded chemotherapy and suspended the use of GnRH-a. 12 months later, a spontaneous pregnancy was normally achieved, which ended with a live newborn at 38.4 weeks of gestation, and 4 years later, a second spontaneous pregnancy, which also culminated in obtaining a healthy newborn. Conclusions: Fertility preservation should be considered a fundamental aspect of the quality of life of cancer survivors. Provided that there are no contraindications, the use of GnRH-a should be considered before the start of chemotherapy, remembering that this treatment could offer benefits beyond the preservation of fertility (bone, sexual, and cardiovascular health), providing comprehensive management.
\end{abstract}

Key words: Gonadotropin-releasing hormone. Fertility preservation. Cancer survivors. Pregnancy.

\section{Introduction}

Cancer incidence increases with time, reaching its peak in people over 50. However, thousands of young women are diagnosed each year ${ }^{1,2}$. Malignant ovarian germ cell tumors account for $<5 \%$ of all ovarian neoplasms, and typically occur in young women, with a higher incidence during reproductive age. They consist of tumors of a histopathological spectrum of primordial germ cells and sexual stroma, with different clinical, biological, and histological features. Approximately $60-70 \%$ of cases are diagnosed in clinical Stage I or II of the International Federation of Gynecology and $\mathrm{Ob}$ stetrics, and $20-30 \%$ in Stage III; a diagnosis in the bilateral involvement or Stage IV is rare. Surgery is the first step in treatment; the information thus provided is essential to determine the spread of the disease, the prognosis, and post-operative management. Fertility-sparing surgery has been done safely and with excellent long-term survival rates, so there is currently a

\section{Correspondence:}

Otto Hugo Valdés-Martínez

E-mail: otto@ottovaldes.com
Available online: 01-10-2018

Date of reception: 12-02-2018

Date of acceptance: 06-03-2018

DOI: 10.24875/RMU.M18000013
Medicina Universitaria. 2018;20(2):84-87 www.medicinauniversitaria.org

作 2017 Universidad Autónoma de Nuevo Lón article under the CC BY-NC-ND license (http://creativecommons.org/licenses/by-nc-nd/4.0/). 
consensus that supports unilateral salpingo-oophorectomy, peritoneal lavage, and careful inspection of the abdominal cavity with proper handling, and preserving reproductive potential. In advanced stages, neoadjuvant chemotherapy is used to increase the probability of success when performing a fertility-sparing surgery, recommended treatment of which consists of 3-4 cycles $^{3}$.

Chemotherapy causes a negative impact on both the function and the ovarian reserve, directly destroying the follicles and accelerating the natural decline that occurs with time ${ }^{4}$. The extent of the damage depends on the age of the patient, the basal ovarian reserve and the type of drug received, as well as the dose and duration. The final effect is variable; in some patients, the menstrual cycles will resume spontaneously, while others may present premature ovarian failure ${ }^{5,6}$.

Direct and indirect mechanisms for the production of ovarian damage have been proposed. Chemotherapeutic agents can cause apoptosis of the follicles, the granular cells in division being particularly susceptible. The loss of follicles in growth causes acceleration in the recruitment of primordial follicles, decreasing the ovarian reserve. The ovarian stroma also shows fibrosis and vascular damage, resulting in local ischemia, contributing to follicular depletion ${ }^{5}$.

The theories that support the use of gonadotropin-releasing hormone agonist ( $\mathrm{GnRH}-\mathrm{a})$ describes both direct and indirect protective effects. $\mathrm{GnRH}-\mathrm{a}$ consists of agonists acting at the level of the anterior pituitary, where they bind to receptors of GnRH. They initially have an effect "on the rise," stimulating the secretion of luteinizing and follicle-stimulating hormone. Prolonged activation of the receptors leads to desensitization and interruption in the pulsatility of $\mathrm{GnRH}$, causing gonadotropin secretion to be regulated downward, causing a state of hypogonadism. Eventually, a decrease in GnRH receptors also occurs. Locally, it is thought that $\mathrm{GnRH}-\mathrm{a}$ decreases vascularity, reducing the concentration of the chemotherapeutic agent that acts directly on the ovary. Furthermore, $\mathrm{GnRH}$-a has been shown to inhibit recruitment of primordial follicles, reducing the acceleration of the depletion of the ovarian reserve and protecting fertility ${ }^{2,5}$. Below is the case of a woman of reproductive age diagnosed with a malignant ovarian tumor, which was handled with $\mathrm{GnRH}-\mathrm{a}$ for the preservation of the ovarian reserve.

\section{Development}

We present a 19-year-old patient who attended consultation for abdominal distention, with a history of
$<3$ years of lower-lip plasty and smoking since the age of 13 at a rate of 3 cigarettes a day. Gynecology history, menarche: 13 years, rhythm 1-8 months for 5-7 days, date of past menstrual period March 2011, cervical cytology denied, beginning of sexual life at the age of 17 , with 2 partners, contraceptive method denied. Present illness began with abdominal distension of 3 weeks of evolution, accompanied by abdominal and colic type of pain, diffuse, of mild intensity, intermittent, with no fever or weight loss. She also developed dysuria, pollakiuria, and tenesmus of 15 days of evolution. Physical examination: vital signs normal, cardiorespiratory with no compromise, abdomen soft, depressible, peristalsis present and ascites fluid without tension, is palpable. Abdominopelvic tumor $18 \mathrm{~cm}$ by $10 \mathrm{~cm}$ in the middle line, defined edges, soft line, without adhesions to deeper planes. Vaginal examination: elastic vagina, yellowish vaginal discharge without the possibility of rating uterine size, cervix $2 \mathrm{~cm}$, without pathological data. Full abdomen contrasted CAT was performed, where the pelvic cavity showed a rounded image, of heterogeneous density, of cyst predominance with partially defined edges, with a measure of $10 \mathrm{~cm} \times 14 \mathrm{~cm} \times 15 \mathrm{~cm}$, which after administering contrast media presented peripheral enhancement, as well as multiple thick septa. The urinary bladder and rectum sigmoid are normal, and we observed copious amounts of ascites. In conclusion, a suggestive image of cystadenocarcinoma within requested laboratory studies: lactic dehydrogenase at $293 \mathrm{IU} / \mathrm{L}$ (91-180 IU/L), alpha-fetoprotein $>1210 \mathrm{ng} / \mathrm{ml}$, and Ca-125 $779.8 \mathrm{U} / \mathrm{ml}$. Due to these findings, she is scheduled for laparotomy with pre-operative diagnosis of right ovarian tumor, probably of a germ source.

On March 15, 2011, a laparotomy was made with a right salpingo-oophorectomy and right pelvic lymphadenectomy, along with an omentectomy, an appendectomy, and resection of the parietal peritoneum; optimal conservative cytoreductive surgery was considered. She stays 3 days in-hospital with proper evolution.

Pathology reported: ascites fluid positive for neoplastic cells, right annex and fragment of omentum: germ mixed tumor $(80 \%$ endodermal sinuses and $20 \%$ embryonal carcinoma) $15.5 \mathrm{~cm}$ in its greater diameter that infiltrates and breaks the capsule with peritoneal implants, oviducts without alterations. Right pelvic lymph nodes tissue free of neoplastic infiltration, omentum fragment with chronic peritonitis and mesothelial hyperplasia. Cecal appendix: endodermal sinus tumor is in the serosa of blood vessels. Parietal peritoneum: germ 
tissue implants. The patient is a candidate for chemotherapy and raises the need to preserve fertility. A central densitometry was done, hormonal profile, lipid profile, and blood chemistry, and began treatment with $\mathrm{GnRH}$ agonists (Lucrin Depot ${ }^{\circledR} 3.75 \mathrm{mg}$ intramuscular) on the $24^{\text {th }}$ day of her cycle, with repetition of the same dose 20 days later.

Studies before the beginning of GnRH agonists: Estradiol $14 \mathrm{pg} / \mathrm{ml}$, follicle stimulating hormone (FSH) $2.6 \mathrm{mUI} / \mathrm{ml}$, total testosterone $0.51 \mathrm{ng} / \mathrm{ml}$, lipid profile, total cholesterol $248 \mathrm{mg} / \mathrm{dl}$, triglycerides $112 \mathrm{mg} / \mathrm{dl}$, high-density lipoprotein $63 \mathrm{mg} / \mathrm{dl}$, low-density lipoprotein $163 \mathrm{mg} / \mathrm{dl}$, glucose $81 \mathrm{mg} / \mathrm{dl}$, creatinine $0.6 \mathrm{mg} / \mathrm{dl}$, bone densitometry, osteopenia in lumbar spine, and normal in the hip.

The patient received chemotherapy with bleomycin, etoposide, and cisplatin administered every 3 weeks for 4 cycles, beginning in May 2011 and ending in August of the same year (Table 1). It was indicated to continue treatment with $\mathrm{GnRH}$ agonists at the same time she was receiving the chemotherapy.

As a side effect of chemotherapy, the patient developed neutropenia Grade 2 without fever, which was resolved in the second cycle. Tumor markers were made after chemotherapy for control: human chorionic gonadotropin beta fraction in $<1.00 \mathrm{mUl} / \mathrm{mL}$, alpha-fetoprotein $3.1 \mathrm{ng} / \mathrm{ml}$, and Ca-125 11.5 U/ml. An imaging CAT with the contrast of abdomen was performed, no tumor recurrence, metastasis or adenopathy was identified. Follow-ups for tumor markers every 2 months and a sectional imaging study every 4 months were ordered.

The past dose of $\mathrm{GnRH}$ agonist was administered on 26/06/2011, presenting spontaneous menstruation in September 2011, requiring a hormone profile and bone densitometry for follow-up, which had a result of FSH $20 \mathrm{mlU} / \mathrm{ml}$. We decided to do a controlled ovarian stimulation due to the spontaneous menstruation for vitrification of oocytes, with an explanation of probable low response.

In July 2012, the patient went to cancer follow-up without identifying tumor recurrence, adenopathy, or metastatic disease. In control laboratory markers: human chorionic gonadotropin beta fraction in $<0.1 \mathrm{mUI} / \mathrm{mL}$ and Ca - $12511.2 \mathrm{U} / \mathrm{ml}$.

The following appointment, $63,367 \mathrm{mUl} / \mathrm{mL}$ of human chorionic gonadotropin beta fraction was detected in the next cancer follow-up. It was a confirmed intrauterine pregnancy, with a normal evolution. A C-section procedure was done in the 38.4 weeks of gestation in May 2013. A female product was obtained, weighing $2910 \mathrm{~g}$, an Apgar of 9/9. During the procedure, a normal left adnexa was observed, peritoneal lavage was done,
Table 1. Chemotherapy treatment schemes administered by cycle

\begin{tabular}{|l|c|l|}
\hline Medication & Dose & Frequency \\
\hline Bleomycin & 30 units & Day 1, day 8 and day 15 \\
\hline Etoposide & $100 \mathrm{mg} / \mathrm{m}^{2}$ & Days $1,2,3,4$ and 5 \\
\hline Cisplatin & $20 \mathrm{mg} / \mathrm{m}^{2}$ & Days $1,2,3,4$ and 5 \\
\hline
\end{tabular}

and a biopsy of bladder and parietal peritoneum were performed. She presented a satisfactory evolution, leaving with her child 2 days after the procedure. The anatomopathological result reported vesicle peritoneum, with edema and vascular congestion, negative for neoplastic cells. Cytology of peritoneal fluid and parietal peritoneum was negative for neoplastic cells. 4 years later, she presented a second spontaneous pregnancy which evolved normally, and a male product of $3080 \mathrm{~g}$, an Apgar of 9/10 was obtained by C-section, with proper postoperative evolution was obtained by a $\mathrm{C}$-section. The patient was discharged from the cancer follow-up without evidence of relapse.

\section{Conclusions}

The cure rate in patients diagnosed with early-stage germ cell malignant ovarian tumors is $100 \%$; in advanced cases, it is $75 \%{ }^{2}$. This case exemplifies the success of therapy combined with surgery and chemotherapy. These advances in treatment and clinical outcomes have brought an increase in interest in preventing long-term complications and improving the quality of life 4 . This, coupled with the current trend of postponing motherhood, has caused an increase in the number of patients who are interested in the preservation of fertility. Our patient is an example of the above since she has had two healthy children even after having undergone chemotherapy for curative purposes. Population with similar characteristics of our patient presents unique medical and psychosocial needs, different from elderly patients who are diagnosed with cancer. This emphasizes the importance of a multidisciplinary approach in its treatment, highlighting the importance of addressing the issue of the preservation of fertility and protection of gonadal function with the patient before the beginning of chemotherapy ${ }^{3,5}$.

Current evidence suggests that the use of $\mathrm{GnRH}-\mathrm{a}$ should be considered in all women of reproductive age who are to receive chemotherapy, provided there are no contraindications since this treatment could offer benefits beyond the preservation of fertility (bone, sexual, and cardiovascular health), providing a comprehensive 
management ${ }^{3,7}$. Cancer can be cured in many instances, so the quality of later life is currently a priority.

\section{Ethical disclosures}

Protection of human and animal subjects. The authors declare that no experiments were performed on humans or animals for this study.

Confidentiality of data. The authors declare that they have followed the protocols of their work center on the publication of patient data.

Right to privacy and informed consent. The authors have obtained the written informed consent of the patients or subjects mentioned in the article. The corresponding author is in possession of this document.

\section{Conflicts of interest}

Authors report no conflics of interest.

\section{Funding}

Authors report no funding.

\section{References}

1. Lambertini M, Del Mastro L, Pescio MC, et al. Cancer and fertility preservation: international recommendations from an expert meeting. BMC Med. 2016;14:1

2. David A, Green LJ, Shikanov A. Fertility preservation in 2016: where are we? Semin Reprod Med. 2017:35:160-6.

3. Di Tucci C, Casorelli A, Morrocchi E, et al. Fertility management for malignant ovarian germ cell tumors patients. Crit Rev Oncol Hematol. 2017;120:34-42.

4. Villarreal-Garza C, Martinez-Cannon BA, Platas A, et al. Fertility concerns among breast cancer patients in mexico. Breast. 2017;33:71-5.

5. Hickman LC, Llarena NC, Valentine LN, Liu X, Falcone T. Preservation of gonadal function in women undergoing chemotherapy: a systematic review and meta-analysis of the potential role for gonadotropin-releasing hormone agonists. J Assist Reprod Genet. 2018;35:571-81.

6. Silva C, Caramelo O, Almeida-Santos T, Ribeiro Rama AC. Factors associated with ovarian function recovery after chemotherapy for breast cancer: a systematic review and meta-analysis. Hum Reprod.2016;31: 2737-49.

7. Moore HC, Unger JM, Phillips KA, et al. Goserelin for ovarian protection during breast-cancer adjuvant chemotherapy. N Engl J Med. 2015;372: 923-32. 